\title{
Predominant role of vasoconstrictors over dilatators derived from arachidonic acid in hypoxic pulmonary vasoconstriction
}

\author{
YESHAN HAN $^{1}$, GAOLIANG YAN ${ }^{2}$, QINGJIE WANG ${ }^{2}$, GENGSHAN MA ${ }^{2}$, \\ CHENGCHUN TANG ${ }^{2}$, YUCHUN GU ${ }^{3}$, LI LI $^{1}$ and JIANZHONG ZHAO ${ }^{4}$ \\ ${ }^{1}$ Department of Anesthesiology, Changzhou No. 2 People's Hospital, Changzhou, Jiangsu 213003; \\ ${ }^{2}$ Department of Cardiology, Zhongda Hospital of Southeast University Medical School, Nanjing, Jiangsu 210009; \\ ${ }^{3}$ Institute of Molecular Medicine, Peking University, Beijing 100871; ${ }^{4}$ Department of Cardiology, \\ Changzhou No. 2 People's Hospital, Changzhou, Jiangsu 213003, P.R. China
}

Received November 26, 2012; Accepted August 8, 2013

DOI: $10.3892 / \mathrm{mmr} .2013 .1645$

\begin{abstract}
Prostanoids derived from arachidonic acid (AA) have been shown to play a permissive role in the regulation of vascular tone and wall tension. Conventionally, epoxyeicosatrienoic acids (EETs) and prostacyclin have been considered as dilatators, whereas thromboxane (TX) and hydroxyeicosatetraenoic acid (HETE) were considered as vasoconstrictors. However, the role of these prostanoids in the mediation of acute hypoxic pulmonary vasoconstriction is not yet clearly understood. In the present study, the role of prostanoids in the acute hypoxic response in rat isolated intrapulmonary arteries (IPAs) was investigated. Exogenous AA directly caused vasoconstriction, but exerted a significant inhibition on hypoxic vasoconstriction. The vasoconstriction by AA was mediated by the endothelium. AA metabolites from lipoxygenase (LOX) had no effect on vascular tone or hypoxic vasoconstriction. Consistent results from the blockage of cytochrome P450 (CYP) or CYP epoxide hydrolase showed that HETE contributed to endothelium-independent hypoxic vasoconstriction. EET via epoxygenase exerted no effect on 80 mM KPSS-induced vessel contraction or hypoxic vasoconstriction. In addition, prostacyclin also failed to inhibit hypoxic pulmonary vasoconstriction (HPV). However, blockage of thromboxane A2/prostanoid (TP) receptors almost eliminated hypoxic vasoconstriction, suggesting the primary role of TP receptors in the regulation of the hypoxic response in rat IPAs. In conclusion, the current data indicate the predominant role of vasoconstrictors instead of dilatators in mediating HPV. These data also highlight a pivotal role for voltage-independent $\mathrm{Ca}^{2+}$ entry in pulmonary hypoxic response and suggest that
\end{abstract}

Correspondence to: Dr Jianzhong Zhao, Department of Cardiology, Changzhou No. 2 People's Hospital, 29 Xing Long Street, Changzhou, Jiangsu 213003, P.R. China

E-mail:ycgu@pku.edu.cn

Key words: hypoxic pulmonary vasoconstriction, intrapulmonary arteries, arachidonic acid modulation of these channels by prostanoids underlies their regulatory mechanisms.

\section{Introduction}

Hypoxic pulmonary vasoconstriction (HPV) is the rapid, reversible increase in pulmonary vascular resistance which occurs when alveolar oxygen tension falls below a threshold level (1). HPV is restricted to segments of the vasculature serving the hypoxic lobe, thereby achieving ventilation/perfusion matching and optimizing systemic $\mathrm{PO}_{2}$ without significantly elevating pulmonary artery pressure. However, a previous study (2) observed that in isolated intrapulmonary arterial rings, HPV is biphasic, consisting of an immediate, endothelium-independent constriction, which peaks in $\sim 10$ min (phase I) and a second, slowly developing endothelium-dependent sustained contraction that peaks at $\sim 40 \mathrm{~min}$ (phase II). Pulmonary arteries receive the most attention in respect to HPV. Sustained HPV eventually leads to pulmonary hypertension, which is a progressive disease of pulmonary vasculature, characterized by elevation of pulmonary artery pressure and resistance. The disease has a short life expectancy and severely affects life quality.

Among the variety of signal transduction pathways in HPV, arachidonic acid (AA) and its metabolites are involved in the maintenance of vascular homeostasis, including vascular tone and vessel wall tension (3). In addition to classic prostanoids derived from cyclooxygenase (COX) and lipoxygenase (LOX), prostanoids derived from the monooxygenase pathway have been receiving increasing attention. Under physiological conditions, epoxyeicosatrienoic acids (EETs), which are produced by the cytochrome P450 (CYP) epoxygenase pathway of AA metabolism and hydroxyeicosatetraenoic acid (HETE), which is derived from hydroxygenase, have been suggested to mediate pulmonary circulation and play a key role in pulmonary vascular remodelling specifically under chronic hypoxic stimulation (4,5). Selective epoxygenase inhibitors significantly reduce acute hypoxic pulmonary vasoconstriction and chronic hypoxia-induced pulmonary vascular remodelling (4). The soluble epoxide hydrolase inhibitor enhances the hypoxic vasoconstriction. However, experiments in isolated perfused 
lungs and in vivo animal models have generated conflicting results. The aforementioned complex systems contain blood cells, connective tissue and interstitial cells, all of which may produce extra and unpredictable prostanoids. Therefore, the results are likely to be complex and difficult to interpret.

In the current study, isolated rat intrapulmonary arteries (IPAs) were used to investigate the role of AA and its metabolites via CYP on basal vascular tone and hypoxic vasoconstriction. The study aimed to understand the contribution of various prostanoids and the activation of their receptors in an acute hypoxic response in rat IPAs.

\section{Materials and methods}

Ethics statement. Animal experiments conformed to the Guide for the Care and Use of Laboratory Animals published by the US National Institutes of Health (DHWE publication no. 96-01, revised in 2002) and were approved by the Ethics Review Board for Animal Studies of the Institute of Molecular Medicine, Peking University (Beijing, China).

Isolated rat IPAs. Male Wistar rats (250-350 g) were anesthetized with sodium pentobarbital and sacrificed by cervical dislocation. All preparations were performed in ice-cold physiological salt solution (PSS) containing: $\mathrm{NaCl}, 118 \mathrm{mM}$; $\mathrm{NaHCO}_{3}, 24 \mathrm{mM} ; \mathrm{MgSO}_{4}, 1 \mathrm{mM} ; \mathrm{NaH}_{2} \mathrm{PO}_{4}, 0.435 \mathrm{mM}$; glucose, $5.56 \mathrm{mM}$; $\mathrm{CaCl}_{2}, 1.8 \mathrm{mM}$ and $\mathrm{KCl}, 4 \mathrm{mM}$. Small IPAs $(150-350 \mu \mathrm{m})$ were isolated from surrounding tissue, cut as rings and mounted in a temperature-controlled myograph system (model: 610M; Danish Myo Technology, Gainesville, FL, USA). Briefly, a segment of rat small IPAs ring, 2-mm long, corresponding to a third or fourth order branch from the IPA, was mounted in a small vessel myograph. Two $40-\mu \mathrm{m}$ wires were threaded through the lumen of the vessel segment. One wire was attached to a stationary support driven by a micrometer, while the other was attached to an isometric force transducer. Vessels were allowed to equilibrate under zero force for $30 \mathrm{~min}$. Prior to experiments, mounted rings were gassed with $95 \%$ air $/ 5 \% \mathrm{CO}_{2}(\mathrm{pH} 7.4)$ at $37^{\circ} \mathrm{C}$ for $1 \mathrm{~h}$ and were maintained with continuous bubbling with this gas during experiments unless otherwise stated. Each ring was initially stretched to give an optimal pressure of $30 \mathrm{mmHg}$. Denuded arteries were obtained by passing through the artery with surgical thread. The condition of endothelium cells was examined by relaxation in response to $1 \mu \mathrm{M}$ acetylcholine (Ach).

Hypoxic instructions. The hypoxic instructions employed in the present study have been described previously (6). Briefly, IPAs were exposed to high KPSS 3 times, which contained: $\mathrm{NaCl}, 42 \mathrm{mM} ; \mathrm{NaHCO}_{3}, 24 \mathrm{mM} ; \mathrm{MgSO}_{4}, 1 \mathrm{mM} ; \mathrm{NaH}_{2} \mathrm{PO}_{4}$, $0.435 \mathrm{mM}$; glucose, $5.56 \mathrm{mM} ; \mathrm{CaCl}_{2}, 1.8 \mathrm{mM}$ and $\mathrm{KCl}$, $80 \mathrm{mM}$, with a $2 \mathrm{~min}$ duration and a $10 \mathrm{~min}$ interval. PGF2 $\alpha$ $(5 \mu \mathrm{M})$ (a higher concentration was required when specific chemicals were used) was added to the solution to achieve a pretone equivalent to $\sim 15 \%$ of KPSS response. When the pretone was stable, IPAs were exposed to a continuous gas of $95 \% \mathrm{~N}_{2} / 5 \% \mathrm{CO}_{2}$ for $40 \mathrm{~min}$, washed with PSS and returned to normoxic conditions. Oxygen levels were monitored using a dissolved oxygen meter in all experiments: at hypoxic conditions, $\sim 2 \% \mathrm{O}_{2}$ and normoxic, $\sim 19 \% \mathrm{O}_{2}$. Reproducible results

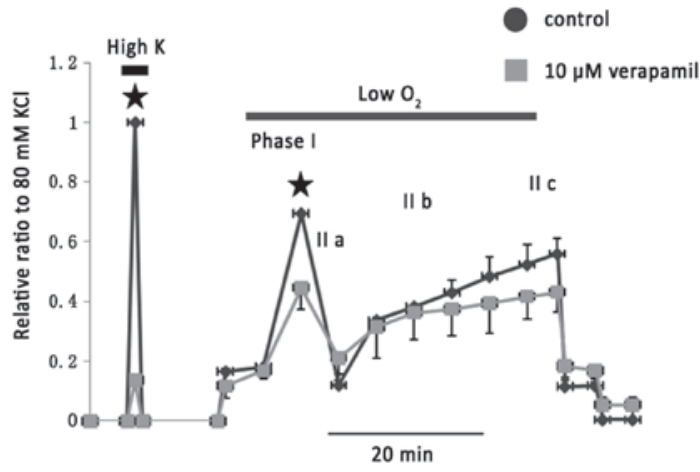

Figure 1 . Inhibitory effect of $10 \mu \mathrm{M}$ verapamil on hypoxic vasoconstriction in isolated rat IPAs. The results demonstrated that $10 \mu \mathrm{M}$ verapamil inhibited $80 \mathrm{mM}$ KPSS-induced vessel constriction to $13.7 \pm 1 \%$ of the control and caused inhibition on phase I of hypoxic vasoconstriction. Symbols represent the mean \pm SEM of 5 IPAs. ${ }^{*} \mathrm{P}<0.05$, vs. control group. IPAs, intrapulmonary arteries.

were obtained following 60-90 min recovery time following exposure to hypoxia, so a standard 80 min recovery was observed between control and testing experiments.

Chemicals. All chemicals were purchased from Biomol International (Plymouth Meeting, PA, USA), with the exception of verapamil (Sigma-Aldrich, St. Louis, MO, USA) and NS398 (Calbiochem, San Diego, CA, USA). They were dissolved in ethanol, dimethyl sulfoxide (DMSO) or distilled water as a stock solution, according to the manufacturer's instructions. Chemicals were diluted to determined concentrations on the date of experiments. Ethanol and DMSO, at the same dilution concentration, were tested and neither vasoconstriction or vasodilation was observed.

Calculations and statistical analysis. All results were normalized using max vasoconstriction induced by $80 \mathrm{mM}$ high $\mathrm{K}$ in the control group. Mean data presented in the histogram is expressed in terms of percentage change in comparison with corresponding parts in the control group. Results are shown as mean \pm SEM and comparisons between groups were performed by paired Student's t-test. All data analysis was performed using SPSS version 13.0 software (SPSS, Inc., Chicago, IL, USA). P $<0.05$ (two-tailed) was considered to indicate a statistically significant difference.

\section{Results}

Exogenous application of AA causes vasoconstriction and attenuates hypoxic vasoconstriction in isolated rat IPAs. IPAs were equilibrated with 2-4 exposures to $80 \mathrm{mM}$ KPSS for a 5 min duration. Thereafter, IPA was pre-contracted with $3 \mu \mathrm{M}$ PGF $2 \alpha$ or $30 \mathrm{mM}$ KPSS to facilitate the hypoxic response. Subsequently, a hypoxic challenge for $45 \mathrm{~min}$ was applied and thereafter washed off. An interval of 60-90 min was employed between 2 hypoxic stimulations to allow producible hypoxic vasoconstriction. Tested chemicals were applied during the 2nd hypoxic challenge. As shown in Fig. 1, $10 \mu \mathrm{M}$ verapamil greatly attenuated $80 \mathrm{mM}$ KPSS-induced vessel contraction and caused an inhibition on phase I of the hypoxic vasoconstriction by $36.7 \pm 5.9 \%(n=5)$. 
A
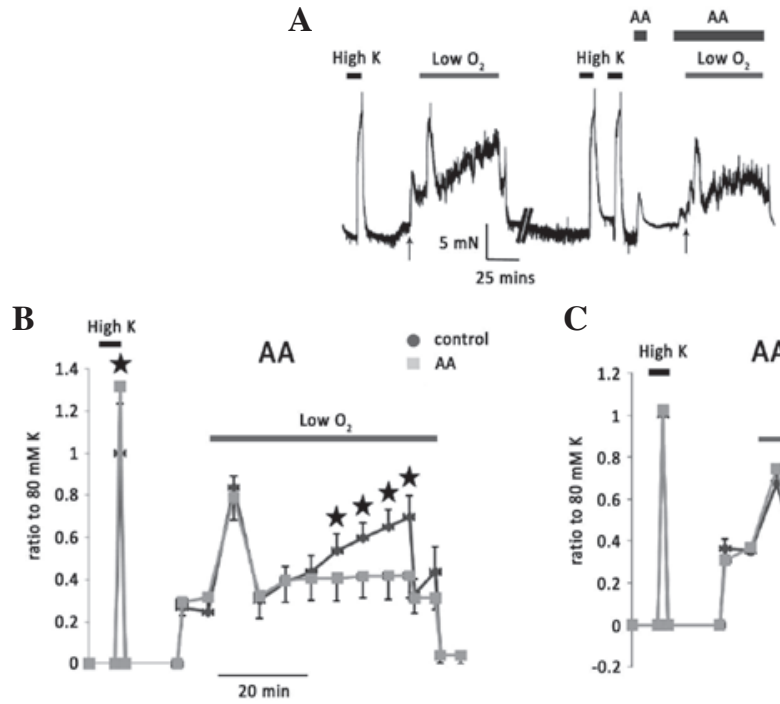

C

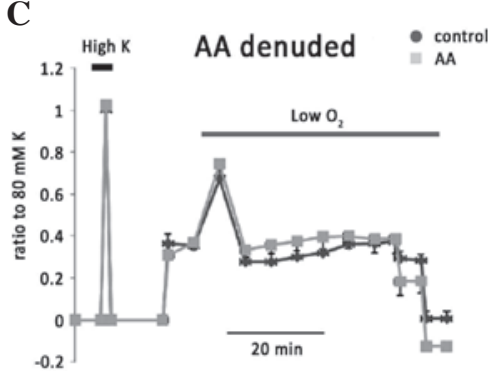

Figure 2. Effects of AA $(20 \mu \mathrm{M})$ on basal tone and hypoxic vasoconstriction of rat IPAs. (A) An example trace showed the effect of AA basal tone and hypoxic vasoconstriction of rat IPAs. (B) AA $(20 \mu \mathrm{M})$ significantly enhanced the $80 \mathrm{mM}$ KPSS-induced vessel contraction, but attenuated phases IIb and IIc of hypoxic vasoconstriction of rat IPAs. (C) AA $(20 \mu \mathrm{M})$ exerted no effect on $80 \mathrm{mM}$ KPSS- or hypoxia-induced vessel contraction in denuded IPAs. "P $<0.05$, vs. control group. AA, arachidonic acid; IPAs, intrapulmonary arteries.
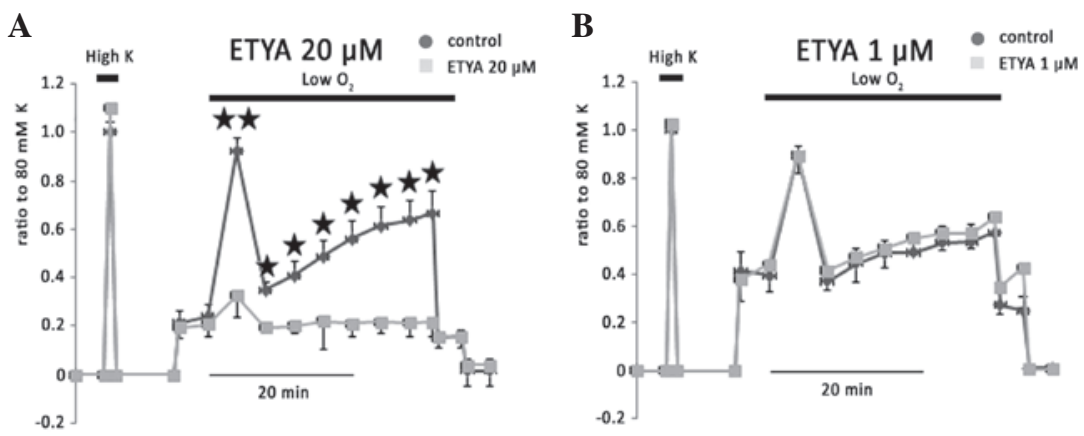

Figure 3. Effects of ETYA on vasoconstriction of IPAs. (A) ETYA ( $20 \mu \mathrm{M})$ slightly but did not significantly enhance 80 mM KPSS-induced vessel contraction and almost eliminated hypoxic vasoconstriction in phase I and II. (B) ETYA $(1 \mu \mathrm{M})$, exerted no effect on $80 \mathrm{mM}$ KPSS- or hypoxia-induced vessel contraction. ${ }^{*} \mathrm{P}<0.05$, vs. respective control group. ETYA, 5,8,11,14-eicosatetraynoic acid; IPAs, intrapulmonary arteries.

Following the same procedure, the exogenous application of AA $(20 \mu \mathrm{M})$ caused a significant transient vasoconstriction. The example trace is shown in Fig. 2A. The basal tension returned to control level when AA was washed off. Phase II of hypoxic vasoconstriction was significantly attenuated by the application of AA (Fig. 2B). In the denuded isolated rat IPAs, $20 \mu \mathrm{M}$ exerted no effect on basal tension or hypoxic vasoconstriction (Fig. 2C), suggesting that endothelium factors are involved in these AA effects.

It is well established that $\mathrm{Ca}^{2+}$ entry via voltage-gated and voltage-independent $\mathrm{Ca}^{2+}$ channels mediates hypoxic vasoconstriction. AA and its metabolites have been observed to directly regulate these $\mathrm{Ca}^{2+}$ channels in an enhancement or inhibition manner (7-9). 5,8,11,14-Eicosatetraynoic acid (ETYA) is a COX and LOX pathway inhibitor. ETYA $(20 \mu \mathrm{M})$, which is resistant to AA degenerative enzymes, had no effect on basal tension and $80 \mathrm{mM}$ KPSS-induced vessel contraction, but greatly inhibited phases I, IIa, IIb and IIc of hypoxic vasoconstriction to $13.1 \pm 7.1,9.3 \pm 14.5,4.9 \pm 3.7$ and $4.3 \pm 4.8 \%$ of controls, respectively (Fig. 3A). This inhibition by ETYA may be due to its blockage effects on COX and CYP. Accordingly,
$1 \mu \mathrm{M}$ ETYA, which is hypothesized to inhibit 12-LOX and 15-LOX, exerted no effect on hypoxic vasoconstriction (Fig. 3B), implying the key role of COX in the regulation of rat IPA vascular wall tension.

Effect of EET and HETE on vasoconstriction of rat IPAs. ETYA $(20 \mu \mathrm{M})$ and AA $(20 \mu \mathrm{M})$ eliminated hypoxic vasoconstriction (Fig. 4A). However, $20 \mu$ M ETYA reversed AA induced vasoconstriction (data not shown), suggesting that AA metabolites generated from endothelium may alter basal tension $(\mathrm{n}=5)$. EET, which is an endothelium-derived relaxing substance and generated via epoxygenase (10) and HETE, which is generated via epoxide hydrolase and contributes vasoconstriction and hypertension (11), have been previously shown to be associated with hypoxic pulmonary hypertension. CYP epoxygenase derived EET is considered to elicit pulmonary vasoconstriction. Therefore, the effect of AA metabolites via CYP on vasoconstriction of IPAs were examined. SKF525a, a general inhibitor of CYP, attenuated $80 \mathrm{mM}$ KPSS-induced vessel contraction to $11.5 \pm 5.4 \%$ of controls and inhibited phase I hypoxic vasoconstriction to $36.4 \pm 5.6 \%$ of 


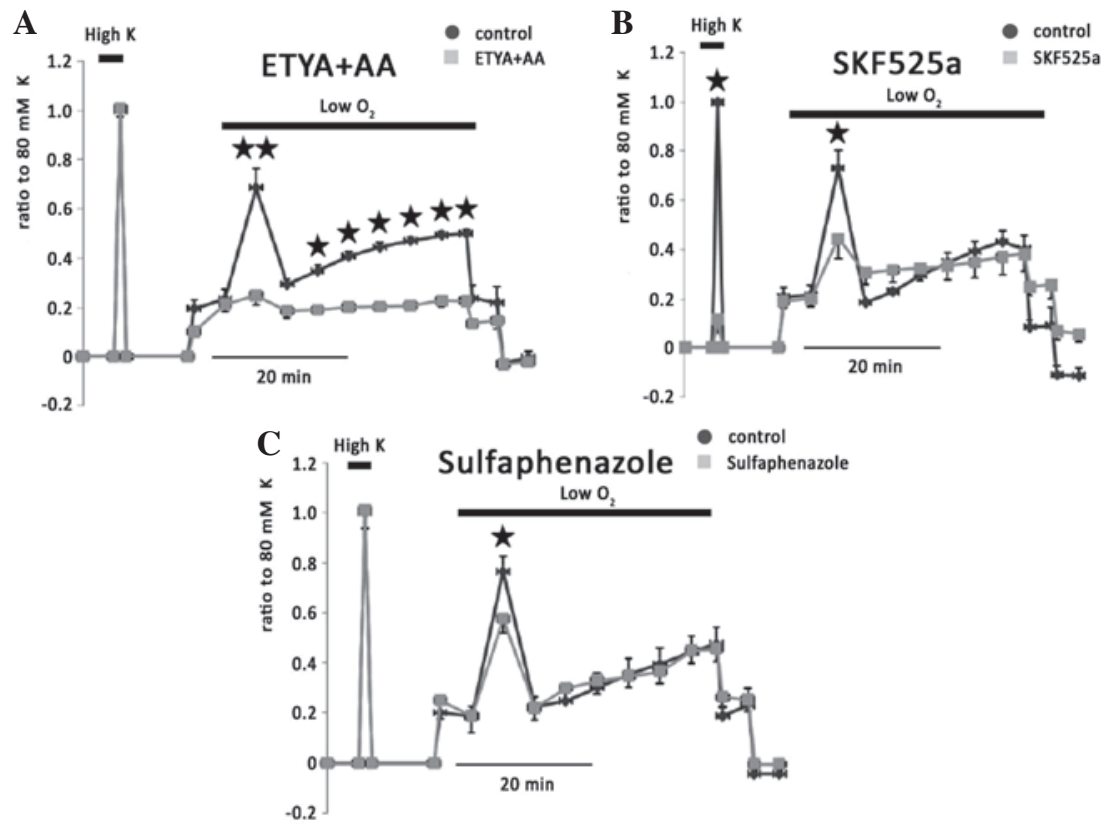

Figure 4. Effects of blocking CYP 450 on vasoconstriction of IPAs. (A) Co-application of $20 \mu \mathrm{M}$ ETYA and $10 \mu \mathrm{M}$ AA eliminated hypoxic vasoconstriction in phase I and II, but exerted no effect on $80 \mathrm{mM}$ KPSS-induced vessel contraction. (B) SKF525a markedly attenuated $80 \mathrm{mM}$ KPSS-induced vessel contraction and exerted a significant inhibition on phase I of hypoxic vasoconstriction. (C) Sulfaphenazole inhibited phase I of hypoxic vasoconstriction. "P $<0.05$, vs. respective control group. CYP 450, cytochrome P450; IPAs, intrapulmonary arteries; ETYA, 5,8,11,14-eicosatetraynoic acid; AA, arachidonic acid.

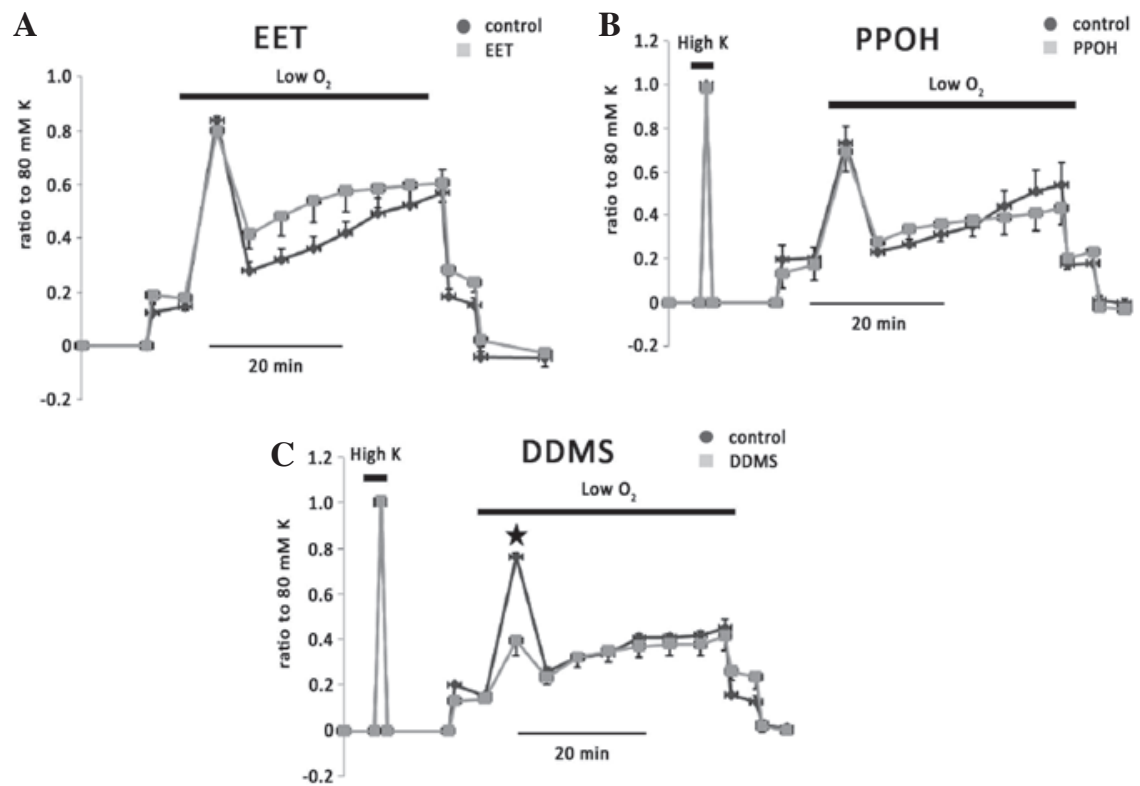

Figure 5. Effects of EET and HETE on vasoconstriction of IPAs. (A) EET (200 nM) only reduced phase I of hypoxic vasoconstriction. (B) Blockage of CYP epoxygenase by PPOH exerted no significant effect on $80 \mathrm{mM}$ KPSS- or hypoxia-induced vessel contraction. (C) Blockage of CYP LOX by DDMS significantly attenuated phase I of hypoxic vasoconstriction. " $\mathrm{P}<0.05$, vs. respective control group. EET, epoxyeicosatrienoic acid; HETE, hydroxyeicosatetraenoic acid; IPAs, intrapulmonary arteries; CYP, cytochrome P450; PPOH, 6-(2-proparglyloxyphenyl)hexanoic acid; LOX, lipoxygenase; DDMS, 12,12-dibromo-N-(methylsulfonyl)-11-dodecenamide.

controls (n=6; Fig. 4B). A possible explanation for the effect of SKF525a may be due to the blockage of $\mathrm{Ca}^{2+}$ channels. To further confirm the role of CYP, sulfaphenazole, another antagonist of CYP, significantly reduced phase I of hypoxic vasoconstriction to $64.9 \pm 25.9 \%$ of controls $(n=6)$, but had no effect on $80 \mathrm{mM}$ KPSS-induced vessel contraction (Fig. 4C).

11,12-EET (200 nM), a major product from CYP 2C9 (12), exerted no effect on hypoxic vasoconstriction (Fig. 5A; $n=3$ ). 6-(2-proparglyloxyphenyl)hexanoic acid (PPOH), an antagonist of CYP epoxygenase, exerted no effect on hypoxic vasoconstriction and $80 \mathrm{mM} \mathrm{KPSS}$-induced vessel contraction (Fig. 5B; $\mathrm{n}=3$ ). 12,12-dibromo-N-(methylsulfonyl)-11-dodecenamide (DDMS), an antagonist of CYP hydroxygenase, significantly blocked phase I of hypoxic vasoconstriction by $58 \pm 11 \%(\mathrm{n}=3$; Fig. 5C), suggesting an involvement of 20-HETE in the hypoxic response in isolated rat IPAs and independence to endothelium. 

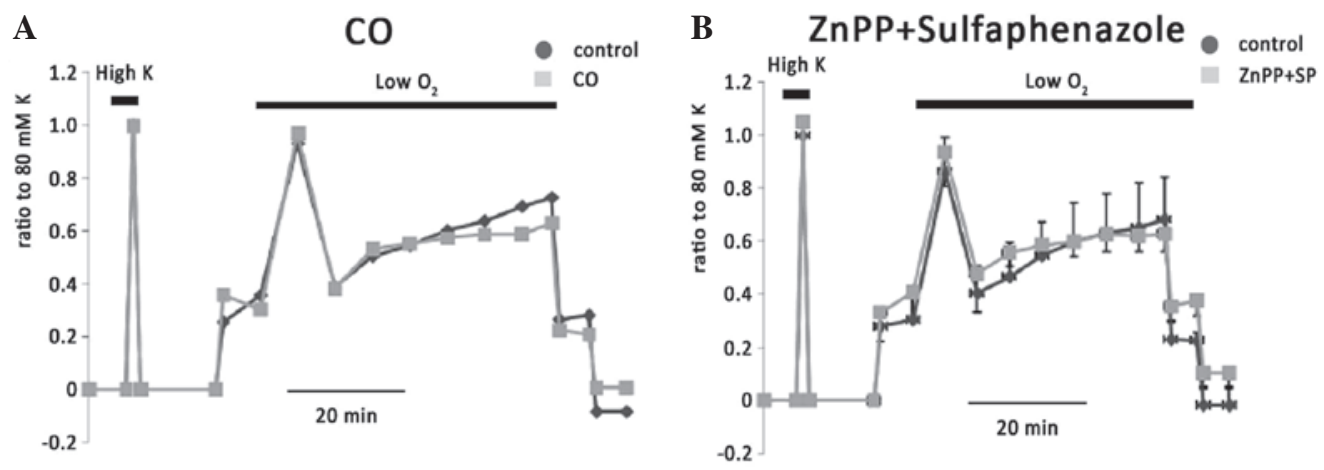

Figure 6. Effects of $\mathrm{CO}$ on vasoconstriction of IPAs. (A) CO had no effect on KPSS-induced vessel contraction or hypoxic vasoconstriction. (B) ZnPP, combination with sulfaphenazole, also had no effect on KPSS-induced vessel contraction or hypoxic vasoconstriction. CO, carbon monoxide; IPAs, intrapulmonary arteries.
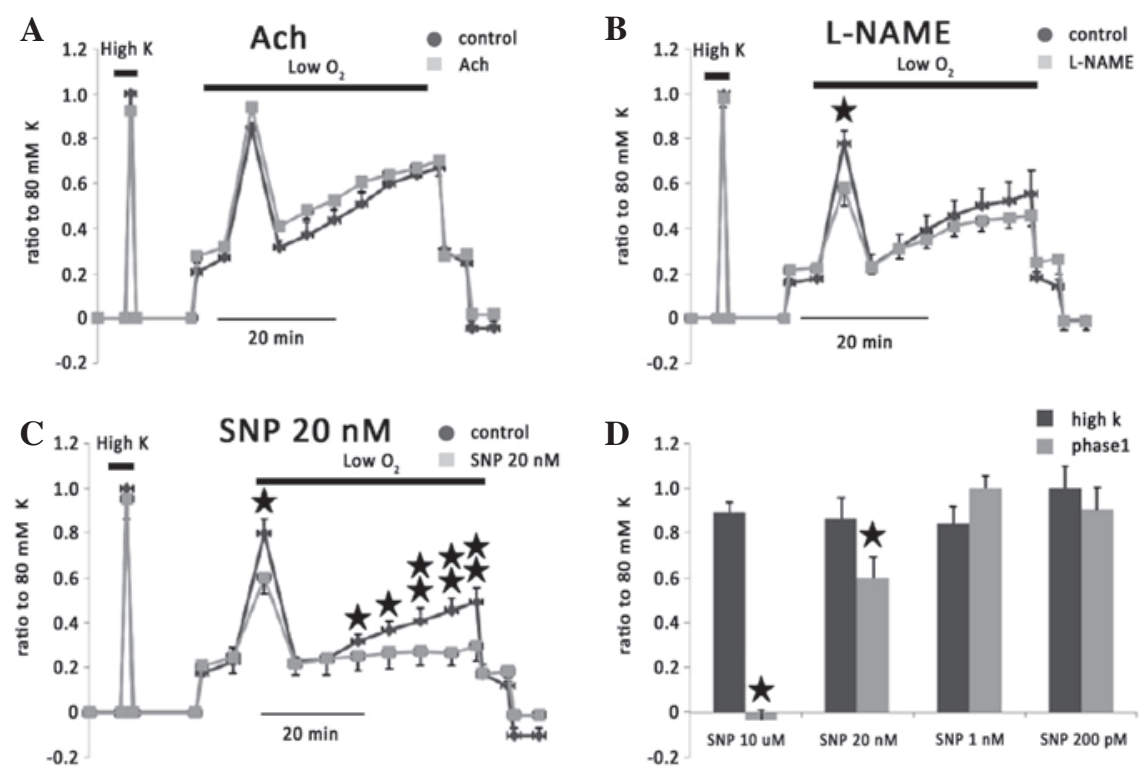

Figure 7. Effect of NO on vasoconstriction of rat IPAs. (A) Ach alone, which induces NO release from endothelium, did not inhibit HPV but exerted a slight facilitate HPV. (B) L-NAME, significantly inhibited phase I of HPV. (C) SNP (20 nM), as a NO donor, significantly inhibited phase I and II of HPV. (D) A variety of concentrations of SNP affect phase I of hypoxia vasoconstriction. "P $<0.05$, vs. respective control group. NO, nitric oxide; IPAs, intrapulmonary arteries; HPV, hypoxic pulmonary vasoconstriction; SNP, sodium nitroprusside; Ach, acetylcholine.

In addition, previous studies have suggested that products of heme oxygenase and CYP are required to attenuate vasoconstriction reactivity (13). Carbon monoxide (CO) has been shown to cause vasoconstriction in pulmonary arteries. $\mathrm{CO}$ and biliverdin are the general products of heme oxygenase. A $\mathrm{CO}$ donor was therefore employed in the current study. $\mathrm{CO}$ alone $(n=6)$, had no effect on KPSS-induced vessel contraction or hypoxic vasoconstriction (Fig. 6A). The combination of $\mathrm{ZnPP}$, which blocks heme oxygenase and sulfaphenazole $(\mathrm{n}=5)$ also had no effect (Fig. 6B).

It has been observed that EET may upregulate nitric oxide (NO) synthase and release (14), resulting in vasodilatation (15). The NO effect on HPV was investigated. Ach alone, which induces NO release from endothelium, did not inhibit HPV but exerted a slight facilitate HPV (Fig. 7A). L-NAME, which is a synthase inhibitor of NO, significantly inhibited phase I of HPV (Fig. 7B). Sodium nitroprusside (SNP; $20 \mathrm{nM}$ ), as a NO donor, significantly inhibited phase I and II of HPV as predicted (Fig. 7C and D).
Effects of prostacyclin and thromboxane $(T X)$ on the vasoconstriction of IPAs. The smaller effect of EET on HPV suggested predominant contraction during the hypoxic condition. Thus, another potent vasodilator, $\mathrm{PGI}_{2}$, which is also metabolized from AA was investigated. $\mathrm{PGI}_{2}$, which is biosynthesized in endothelial cells, exerted no effect on $80 \mathrm{mM}$ KPSS or hypoxic vasoconstriction in isolated rat IPAs ( $n=8$; Fig. 8A). Since its half life under physiological conditions is $2 \mathrm{~min}, \mathrm{PGI}_{2}$ was added to the bath every $2 \mathrm{~min}$ to maintain the concentration during the entire hypoxic challenge. Carbacyclin, which is a stable $\mathrm{PGI}_{2}$, had no effect (Fig. 8B). 15(s)-HPETE, which inhibits prostacyclin synthase, exerted no effect on hypoxic vasoconstriction and 80 mM KPSS-induced vessel contraction (Fig. 8C). However, Forskolin, a potent stimulator of platelet aggregation and release of TX, significantly eliminated hypoxic vasoconstriction in phases I and II (Fig. 8D).

To confirm the hypothesis that vasoconstriction during hypoxia overrides the dilatators, blockage of vasoconstric- 
A

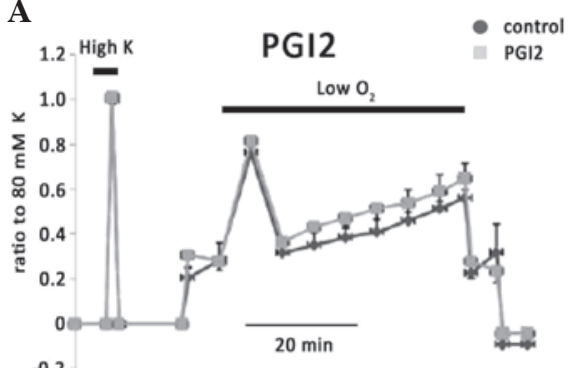

C

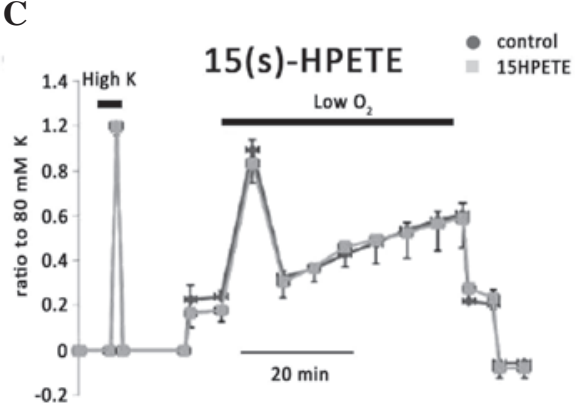

B

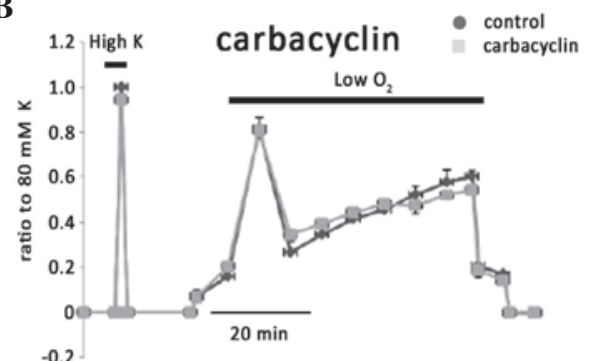

$-0.2$

D

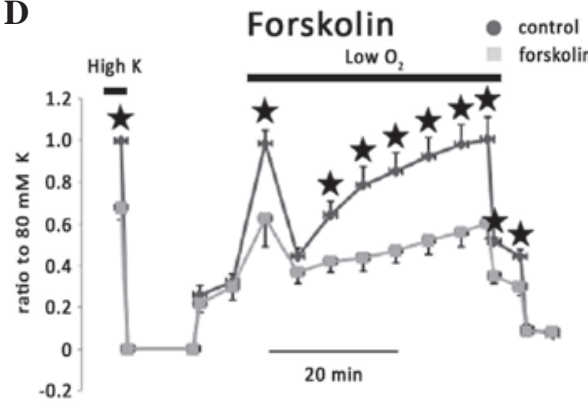

Figure 8. Effect of prostacyclin on vasoconstriction of rat IPAs. (A) Exogenous $\mathrm{PGI}_{2}$ exerted no effect on $80 \mathrm{mM}$ KPSS- or hypoxia-induced vessel contraction. (B) Carbacyclin, which is a stable PGI, also had no effect. (C) 15(s)-HPETE, an inhibitor of prostacyclin synthase, exerted no effect on the vasoconstriction of rat IPAs. (D) Forskolin, a potent stimulator of platelet aggregation and release of TX, significantly eliminated hypoxic vasoconstriction in phases I and II. "P $<0.05$ represents the significant difference. IPAs, intrapulmonary arteries; 15(s)-HPETE, 15S-hydroperoxy-5Z,8Z,11Z,13E-eicosatetraenoic acid; TX, thromboxane.

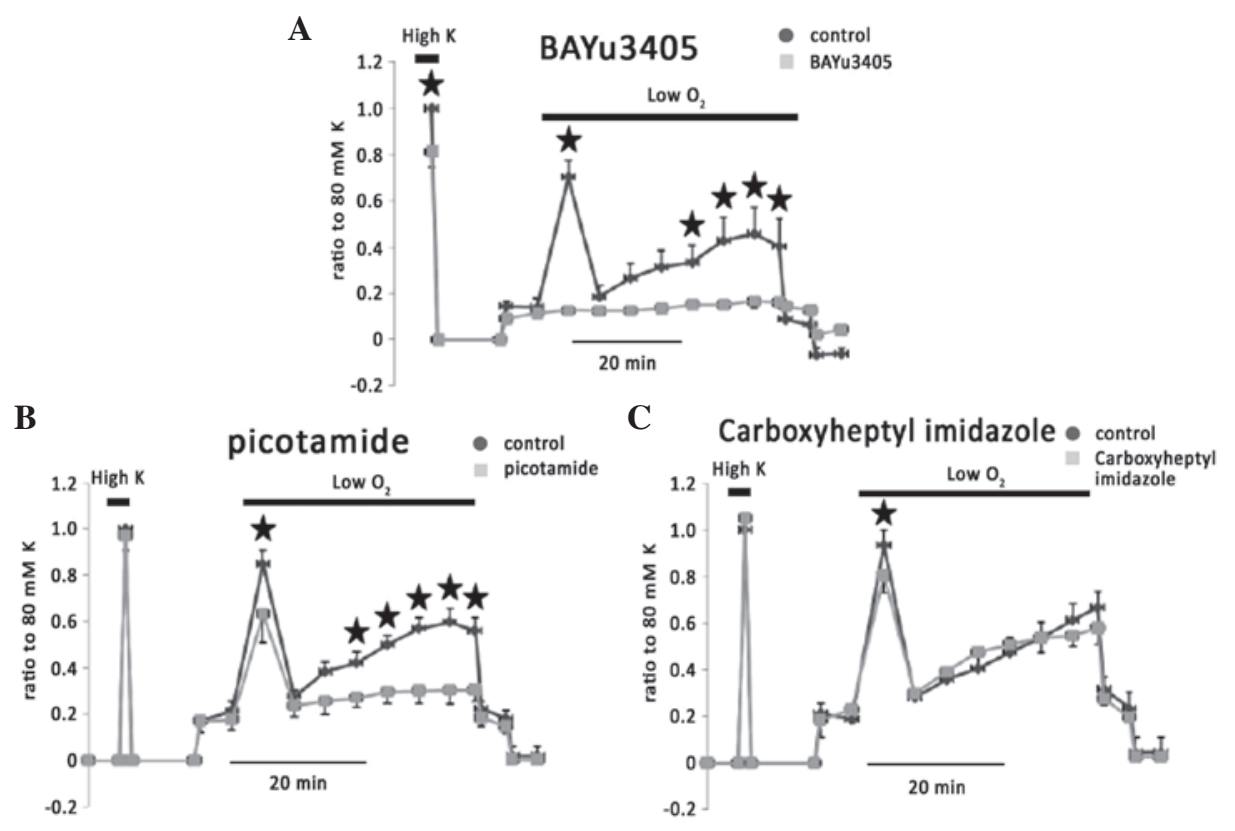

Figure 9. Effect of TX on vasoconstriction of rat IPAs. (A) BAYu3405, an antagonist of the TP receptor, eliminated phase I and II of hypoxic vasoconstriction in rat IPAs. (B) Picotamide, which blocks TXA ${ }_{2}$ activity and inhibits $\mathrm{TXA}_{2}$ synthase, eliminated phase II of hypoxic vasoconstriction and significantly attenuated phase I of hypoxic vasoconstriction. (C) Carboxyheptyl imidazole, an inhibitor of TX synthase, caused an inhibition on phase I of hypoxic vasoconstriction. ${ }^{*} \mathrm{P}<0.05$ represents the significant difference. TX, thromboxane; IPAs, intrapulmonary arteries; TP, thromboxane A2/prostanoid.

tion was employed during a period of hypoxic stimulation. TX is considered as a major vasoconstrictor. Blockage of the TX receptor by BAYu345 significantly inhibited phase I, IIb and IIc of hypoxic vasoconstriction by $78.7 \pm 3.3,79.3 \pm 5.1$ and $74.9 \pm 10 \%$, respectively $(n=5$; Fig. 9A). Picotamide $(100 \mu \mathrm{M})$, which inhibits $\mathrm{TXA}_{2}$ activity and synthase, also inhibited phase I, IIb and IIc of hypoxic vasoconstriction by $18.5 \pm 7.4,57 \pm 8.3$ and $50 \pm 10 \%$, respectively (Fig. 9B; $n=5$ ).
Carboxyheptyl imidazole, which blocks TX synthase reduced phase I of hypoxic vasoconstriction (Fig. 9C).

\section{Discussion}

The present study showed that exogenous AA is capable of eliciting vasoconstriction to enhance the vascular tone via an endothelium-dependent mechanism. In the acute hypoxic 
stimulation, AA metabolites via COX and CYP but not LOX, played a primary role in the formation and regulation of hypoxic vasoconstriction. Although metabolites through CYP only encompass a part of hypoxic vasoconstriction, the pathway via CYP hydroxylase, but not via epoxygenase, is hypothesized to be involved in initiating transient vasoconstriction. In addition, activation of TP receptors is predominant during acute hypoxic challenges. Other prostanoids initiate hypoxic vasoconstriction via TP receptors, since blockage of TX synthesis removes part of hypoxic vasoconstriction. These results determined a principal role of TP receptors in acute hypoxia response and also suggested that vasoconstrictors are significant in forming hypoxic vasoconstriction and that vasodilatators are potent in forming vascular tone.

A previous study on AA metabolites via CYP in pulmonary blood vessels was performed in whole animal (16) or perfused lungs (17). Although the aforementioned experimental conditions are consistent with the physiological environment, it is difficult to determine the contributions between endothelium and smooth muscle. In addition, inflammatory factors and the diverse prostanoids released and generated from blood cells, which are in abundance in an organ, are likely to exert more profound effects on pulmonary blood vessels, resulting in difficulties in interpreting the results. Therefore, isolated IPAs were used in the present study to examine the role of AA and its metabolites on acute hypoxic vasoconstriction. The advantage of the system is to contain simple and minimum tissue for investigation. Although blood vessel rings are stretched by mechanical force and equilibrated with high $\mathrm{K}^{+}$ medium to initiate a basal tone, significant relaxation caused by vasodilatators e.g., Ach and $\mathrm{NO}$ are more evident when vessels are markedly contracted by norepinephrine. However, this relaxation has been investigated in the current study. If the relaxation caused by relaxants in basal tension level was too small to be observed, inhibition on $80 \mathrm{mM}$ KPSS-induced vessel contraction or hypoxic vasoconstriction should aid in understanding the relaxation observed. Therefore, KPSS stimulation was repeated prior to and following application of chemicals. The statistical results on high $\mathrm{K}$ and hypoxia induced vessel contraction were also presented.

Inconsistent with a previous study, AA exerted vasoconstriction in pulmonary artery but not vasodilatation (18). This may be simply due to species and tissue difference. For example, AA causes significant vessel contraction in human pulmonary vein and AA infusion triggers marked, sustained pulmonary vasoconstriction and pulmonary hypertension in broilers (19). The vasoconstriction effect of AA is endothelium dependent, since AA exerts no effect on denuded vessel rings. In addition, this contraction effect is unlikely due to the effect of AA and is mediated by AA metabolites, since $20 \mu \mathrm{M}$ ETYA may reverse this effect. The possible candidates include a number of EETs (20) and 20-HETE (21), which are produced in the endothelium and cause constriction in isolated pulmonary blood vessel rings. In renal and mesenteric arteries, AA induces relaxation through the LOX pathway (22). However, in isolated IPAs, LOX appears to have no effect on basal tone or hypoxic vasoconstriction.

Although ETYA is primarily regarded as a pharmacological analog of AA and resistant to AA degenerative enzymes, a number of other aspects of ETYA were also considered in the present study. ETYA is hypothesized to inhibit AA uptake, COX, all LOXs and CYP. This led to a rational explanation that $20 \mu \mathrm{M}$ ETYA almost eliminated hypoxic vasoconstriction but $1 \mu \mathrm{M}$ ETYA had no effect. This result was further confirmed by blockage of TP receptors, suggesting that prostanoids, via COX and CYP, play a permissive role in mediating the hypoxic response in rat IPAs. The profound effects of ETYA on AA degenerative enzymes have also been observed in the canine femoral artery, in which formation of prostanoids in endothelium was blocked by ETYA, resulting in reversing the AA and Ach relaxant effect.

Prostanoids derived from CYP have been markedly addressed in the accumulating literature as playing a key role in mediating pulmonary vascular tone and wall tension $(4,10,23)$. The results that 11,12-EET activates $\mathrm{Ca}^{2+}$-activated $\mathrm{K}^{+}$channels (BK) (24) and apamin-sensitive $\mathrm{K}^{+}$channels (25) in vascular smooth muscle cells and 20-HETE attenuates BK (26), further highlight the mechanism of degenerative products via CYP in the regulation of blood vessel tone. The current results from sulfaphenazole and DDMS, demonstrate that 20-HETE, via CYP epoxide hydrolase, is involved in hypoxic vasoconstriction and its effect is limited only in part of vessel contraction, which is independent to the endothelium. By comparison with the effect of blockage of the TP receptor, prostanoids via CYP are capable of facilitating a hypoxic response. These results indicate that voltage-gated $\mathrm{Ca}^{2+}$ entry relies on a part of hypoxic vasoconstriction in IPAs and $\mathrm{Ca}^{2+}$ elevation in pulmonary artery smooth muscle cells $(7,27,28)$. Modulation of membrane potential by BK, EET and HETE (29) may therefore, affect a part of endothelium-independent hypoxic vasoconstriction and this is demonstrated in the present study. Activation of TP receptors, which are G-protein coupled receptors, potently modulates voltage-independent $\mathrm{Ca}^{2+}$ entry, including receptor operated $\mathrm{Ca}^{2+}$ entry and store operated $\mathrm{Ca}^{2+}$ entry, resulting in modulation of hypoxic vasoconstriction. $\mathrm{Ca}^{2+}$ entry via voltage-independent pathways appears to play a primary role in the hypoxic response in rat IPAs. The current observations are inconsistent to the previous studies in Webster mice (4), in which selective epoxygenase inhibition significantly reduces hypoxic pulmonary vasoconstriction and chronic hypoxia-induced pulmonary vascular remodeling. The difference, in conclusion, may be due to the difference in the experimental system and observation period. In the previous experiment, the mouse was intermittently exposed to chronic hypoxia. The significant elevations of CYP 2C9 and EET have been revealed by chronic hypoxic stimulation. In the long term, EET modulates membrane potential resulting in alternation in vascular tone and eventual pulmonary hypertension. In the current study, the responses of IPAs were examined. Hypoxic challenge was $<50 \mathrm{~min}$.

The majority of studies on vascular tone describe contractions produced by $\mathrm{TX}$ analogs and relaxations induced by $\mathrm{PGI}_{2}$ analogs (3). A number of vascular smooth muscles express TP and prostacyclin receptors (IP) accordingly. PGI have been found to exhibit potent dilatation in pulmonary arteries (30) via an increase of cellular cAMP. Forsklin markedly inhibits HPV but not $\mathrm{PGI}_{2}$, suggesting a lower density of IP receptors in the pulmonary artery or an unknown mechanism which is required to activate IP. In the present study, the TP receptor has been implicated to mediate hypoxic vasoconstriction in isolate 
rat IPAs. This observation is consistent with previous studies in human pulmonary artery rings. Although BAYu3405 exhibits a strong effect on the prostaglandin D (DP) receptor, a similar blockage effect from picotamide, which is an antagonist of $\mathrm{TXA}_{2}$, confirms the role of TP receptors in hypoxic vasoconstriction. In addition, DP receptors also contribute to part of endothelium independent hypoxic vasoconstriction. There appears to be a minimum of nine $\mathrm{G}$ proteins coupled to TP receptors (31). Although the significance of this promiscuity remains unknown, stimulation of TP receptors leads to activation of phospholipase $\mathrm{C}$, generation of inositol trisphosphate and dystrophin-associated glycoprotein, activation of PKC and myosin light chain kinase, etc. (3), resulting in the regulation of the voltage-independent $\mathrm{Ca}^{2+}$ channel.

Decreased production of NO has been proposed as being significant in the development of pulmonary arterial hypertension. Increased NO formation in the pulmonary circulation exerts a compensatory mechanism to offset the degree of pulmonary vasoconstriction (32). The current results are consistent with these previous observations. SNP blocks hypoxic vasoconstriction. However, controversial negative results have been observed (33), by administration of NO to reduce pulmonary hypertension. A constitutive hyperactivation of NO in caveolin-1-deficient mice causes severe lung fibrosis with marked pulmonary hypertension. Administration of L-NAME attenuates the hypoxic vasoconstriction and relieves pulmonary hypertension (34). L-NAME attenuates NO release and should augment hypoxic vasoconstriction. No effect of L-NAME on HPV from the current results markedly suggested vasoconstrictors as being predominant in HPV. It may also be reflected by the evidence that administration of L-NAME causes a slight increase in tonic pulmonary vascular tone (35) or no alteration in baseline pulmonary arterial pressure during physiological hypoxic response (36).

The present data revealed that exogenous AA exerts vasoconstriction on the basal tone of isolated rat IPAs. This effect is endothelium dependent and may be blocked by antagonists of COX and CYP. The prostanoids derived from CYP hydroygenase, but not from epoxygenase, contribute to hypoxic vasoconstriction in rat IPAs. The activation of $\mathrm{TP}$ receptors plays a permissive role in the regulation of the hypoxic response in rat IPAs.

\section{Acknowledgements}

This study was partly supported by a grant from the Natural Science Foundation of China (grant no. 81170105) to Chengchun Tang (Department of Cardiology, Zhongda Hospital of Southeast University Medical School).

\section{References}

1. Sommer N, Dietrich A, Schermuly RT, et al: Regulation of hypoxic pulmonary vasoconstriction: basic mechanisms. Eur Respir J 32: 1639-1651, 2008.

2. Moudgil R, Michelakis ED and Archer SL: Hypoxic pulmonary vasoconstriction. J Appl Physiol 98: 390-403, 2005.

3. Norel X: Prostanoid receptors in the human vascular wall. ScientificWorldJournal 7: 1359-1374, 2007.

4. Pokreisz P, Fleming I, Kiss L, et al: Cytochrome P450 epoxygenase gene function in hypoxic pulmonary vasoconstriction and pulmonary vascular remodeling. Hypertension 47: 762-770, 2006.
5. Dhanasekaran A, Al-Saghir R, Lopez B, et al: Protective effects of epoxyeicosatrienoic acids on human endothelial cells from the pulmonary and coronary vasculature. Am J Physiol Heart Circ Physiol 291: H517-H531, 2006.

6. Frazziano G, Moreno L, Moral-Sanz J, et al: Neutral sphingomyelinase, NADPH oxidase and reactive oxygen species. Role in acute hypoxic pulmonary vasoconstriction. J Cellular Physiol 226: 2633-2640, 2011.

7. Meng F, To WK and Gu Y: Inhibition effect of arachidonic acid on hypoxia-induced $[\mathrm{Ca}(2+)](\mathrm{i})$ elevation in $\mathrm{PC} 12$ cells and human pulmonary artery smooth muscle cells. Respir Physiol Neurobiol 162: 18-23, 2008.

8. Harteneck C: Function and pharmacology of TRPM cation channels. Naunyn Schmiedebergs Arch Pharmacol 371: 307-314, 2005.

9. Grimm C, Kraft R, Schultz G and Harteneck C: Activation of the melastatin-related cation channel TRPM3 by D-erythro-sphingosine [corrected]. Mol Pharmacol 67: 798-805, 2005.

10. Fleming I: Epoxyeicosatrienoic acids, cell signaling and angiogenesis. Prostaglandins Other Lipid Mediat 82: 60-67, 2007.

11. Llinás MT, Alexander BT, Capparelli MF, Carroll MA and Granger JP: Cytochrome P-450 inhibition attenuates hypertension induced by reductions in uterine perfusion pressure in pregnant rats. Hypertension 43: 623-628, 2004.

12. Larsen BT, Gutterman DD, Sato A, et al: Hydrogen peroxide inhibits cytochrome p450 epoxygenases: interaction between two endothelium-derived hyperpolarizing factors. Circ Res 102: 59-67, 2008.

13. Naik JS and Walker BR: Role of vascular heme oxygenase in reduced myogenic reactivity following chronic hypoxia. Microcirculation 13: 81-88, 2006.

14. Zhang L, Cui Y, Geng B, Zeng X and Tang C: 11,12-Epoxyeicosatrienoic acid activates the L-arginine/nitric oxide pathway in human platelets. Mol Cell Biochem 308: 51-56, 2008.

15. Hercule HC, Schunck WH, Gross V, et al: Interaction between P450 eicosanoids and nitric oxide in the control of arterial tone in mice. Arterioscler Thromb Vasc Biol 29: 54-60, 2009.

16. Gao Y, Feng J, Ma K, et al: 8,9-Epoxyeicosatrienoic acid inhibits antibody production of B lymphocytes in mice. PLoS One 7: e40258, 2012.

17. Kiss L, Schütte H, Padberg W, et al: Epoxyeicosatrienoates are the dominant eicosanoids in human lungs upon microbial challenge. Eur Respir J 36: 1088-1098, 2010.

18. Li Y, Connolly M, Nagaraj C, et al: Peroxisome proliferator-activated receptor- $\beta / \delta$, the acute signaling factor in prostacyclin-induced pulmonary vasodilation. Am J Respir Cell Mol Biol 46: 372-379, 2012.

19. Alvarez-Medina DI, Hernandez A and Orozco C: Endothelial hyperpolarizing factor increases acetylcholine-induced vasodilatation in pulmonary hypertensive broilers arterial rings. Res Vet Sci 92: 1-6, 2012.

20. Liu Y, Wang R, Li J, et al: Stable EET urea agonist and soluble epoxide hydrolase inhibitor regulate rat pulmonary arteries through TRPCs. Hypertens Res 34: 630-639, 2011.

21. Jacobs ER, Bodiga S, Ali I, et al: Tissue protection and endothelial cell signaling by 20-HETE analogs in intact ex vivo lung slices. Exp Cell Res 318: 2143-2152, 2012.

22. Chawengsub Y, Gauthier KM and Campbell WB: Role of arachidonic acid lipoxygenase metabolites in the regulation of vascular tone. Am J Physiol Heart Circ Physiol 297: H495-H507, 2009.

23. Michaelis UR, Xia N, Barbosa-Sicard E, Falck JR and Fleming I: Role of cytochrome P450 2C epoxygenases in hypoxia-induced cell migration and angiogenesis in retinal endothelial cells. Invest Ophthalmol Vis Sci 49: 1242-1247, 2008.

24. Higashimori H, Blanco VM, Tuniki VR, Falck JR and Filosa JA: Role of epoxyeicosatrienoic acids as autocrine metabolites in glutamate-mediated $\mathrm{K}^{+}$signaling in perivascular astrocytes. Am J Physiol Cell Physiol 299: C1068-C1078, 2010.

25. Ayajiki K, Fujioka H, Toda N, et al: Mediation of arachidonic acid metabolite(s) produced by endothelial cytochrome P-450 3A4 in monkey arterial relaxation. Hypertens Res 26: 237-243, 2003

26. Gebremedhin D, Yamaura $K$ and Harder DR: Role of 20-HETE in the hypoxia-induced activation of $\mathrm{Ca}^{2+}$-activated $\mathrm{K}^{+}$channel currents in rat cerebral arterial muscle cells. Am J Physiol Heart Circ Physiol 294: H107-H120, 2008.

27. Robertson TP, Hague D, Aaronson PI and Ward JP: Voltage-independent calcium entry in hypoxic pulmonary vasoconstriction of intrapulmonary arteries of the rat. J Physiol 525: 669-680, 2000 
28. Meng F, To WK and Gu Y: Role of TRP channels and NCX in mediating hypoxia-induced $\left[\mathrm{Ca}^{2+}\right](\mathrm{i})$ elevation in PC12 cells. Respir Physiol Neurobiol 164: 386-393, 2008.

29. Fleming I and Busse R: Endothelium-derived epoxyeicosatrienoic acids and vascular function. Hypertension 47: 629-633, 2006.

30. Morales-Blanhir J, Santos S, de Jover L, et al: Clinical value of vasodilator test with inhaled nitric oxide for predicting long-term response to oral vasodilators in pulmonary hypertension. Respir Med 98: 225-234, 2004.

31. Cyphert JM, Allen IC, Church RJ, et al: Allergic inflammation induces a persistent mechanistic switch in thromboxane-mediated airway constriction in the mouse. Am J Physiol Lung Cell Mol Physiol 302: L140-L151, 2012.

32. Baliga RS, Milsom AB, Ghosh SM, et al: Dietary nitrate ameliorates pulmonary hypertension: cytoprotective role for endothelial nitric oxide synthase and xanthine oxidoreductase. Circulation 125: 2922-2932, 2012.
33. KästnerSB,Kull S,KutterAP,Boller J,Bettschart-WolfensbergerR and Huhtinen MK: Cardiopulmonary effects of dexmedetomidine in sevoflurane-anesthetized sheep with and without nitric oxide inhalation. Am J Vet Res 66: 1496-1502, 2005.

34. Wunderlich C, Schmeisser A, Heerwagen C, et al: Chronic NOS inhibition prevents adverse lung remodeling and pulmonary arterial hypertension in caveolin-1 knockout mice. Pulm Pharmacol Ther 21: 507-515, 2008.

35. Schwenke DO, Pearson JT, Tsuchimochi H, Kangawa K and Shirai M: Pulmonary vascular reactivity of spontaneously hypertensive rats is exacerbated in response to the central administration of exogenous nitric oxide. Clin Exp Pharmacol Physiol 34: 88-94, 2007.

36. Schwenke DO, Pearson JT, Mori H and Shirai M: Does central nitric oxide elicit pulmonary hypertension in conscious rats? Respir Physiol Neurobiol 153: 250-260, 2006. 